\title{
NOTAS NOMENCLATURALES EN EL GÉNERO SCHINOPSIS (ANACARDIACEAE)
}

\author{
VIRGINIA Y. MOGNI ${ }^{1,2}$, DARIÉN E. PRADO ${ }^{1,2}$ y LUIS J. OAKLEY ${ }^{1,3}$
}

\begin{abstract}
Summary: Nomenclatural notes in the genus Schinopsis (Anacardiaceae). During the monographic study of the genus Schinopsis (Anacardiaceae) a nomenclatural revision of its species and synonyms was performed. The names Schinopsis brasiliensis, S. brasiliensis var. glabra, S. heterophylla, S. marginata, and $S$. peruviana are lectotypified, and some cases of inadvertent lectotypifications in previous works are indicated. An epitype for S. haenkeana, entity so far considered doubtful, is designated.
\end{abstract}

Key words: Epitype, lectotype, nomenclature, Schinopsis.

Resumen: Durante el estudio monográfico del género Schinopsis (Anacardiaceae) se realizó una revisión nomenclatural de sus especies y sinónimos. Se lectotipifican los nombres Schinopsis brasiliensis, $S$. brasiliensis var. glabra, S. heterophylla, S. marginata y S. peruviana y se indican algunos casos de lectotipificaciones desapercibidas en trabajos previos. Se designa un epitipo para S. haenkeana, entidad considerada hasta ahora como taxón dudoso.

Palabras clave: Epitipo, lectotipo, nomenclatura, Schinopsis.

\section{INTRODUCCIÓN}

El género Schinopsis Engl. (Anacardioideae, Anacardiaceae, Sapindales; Judd et al., 1999; Pell, 2004) comprende siete especies de árboles neotropicales (Mogni, 2015) cuya distribución geográfica está restringida a los Bosques Secos Estacionales Neotropicales ("BSEN" sensu Prado, 2000) y a los subtropicales chaqueños (Prado, 1993a, b) de Sudamérica. En el contexto de la revisión monográfica del género, se realizó la revisión nomenclatural de todas sus especies (incluidos los sinónimos).

\section{Materiales y Métodos}

Se analizaron los protólogos de los nombres de las especies estudiadas y los ejemplares tipo

\footnotetext{
${ }^{1}$ Botánica, Facultad de Ciencias Agrarias, Universidad Nacional de Rosario, C.C. №14, 2125 Zavalla, Argentina.

${ }^{2}$ Instituto de Investigaciones en Ciencias Agrarias, IICARCONICET, 2125 Zavalla, Argentina.

${ }^{3}$ E-mail: loakley@unr.edu.ar
}

depositados en los siguientes herbarios (Thiers, 2013): BA, BAB, CORD, CTES, FCQ, L, LIL, LP, SI y UNR. También se consultaron las imágenes digitales de materiales tipo disponibles en "JStor Global Plants" (http://www.plants.jstor.org/), así como de fotos digitalizadas de tipos de herbarios europeos realizadas por el Field Museum (https:// www.fieldmuseum.org/node/5186 y http://www. gbif.org/dataset/90c853e6-56bd-480b-8e 8f$6285 \mathrm{c} 3 \mathrm{f} 8 \mathrm{~d} 42 \mathrm{~b})$.

\section{Resultados}

A continuación se listan todos los taxones aceptados para el género Schinopsis, incluidos los sinónimos correspondientes. En cada caso se indica la información nomenclatural y de los ejemplares tipo. La justificación de la designación de lectotipos, epitipos y/o las consideraciones puntuales sobre el status taxonómico de las entidades, se expresan en cada especie.

Schinopsis Engl., en Martius, Fl. Bras. 12(2): 403. 1876. Especie Tipo: Schinopsis brasiliensis 
Engl. (Lectotipo designado por Cabrera, 1938: 50).

= Quebrachia Griseb., Abh. Königl. Ges. Wiss. Göttingen 24: 95. 1879; Symb. Fl. Argent.: 95. 1879. Especie Tipo: Quebrachia lorentzii (Griseb.) Griseb. (三Loxopterygium lorentzii Griseb.).

1. Schinopsis balansae Engl., Bot. Jahrb. Syst. 6: 286. 1885. Tipo: Paraguay. "Bords du Mbay, près de Paraguarí, dans les argiles imperméables", 1-I-1882, Balansa 3188 (Lectotipo G, designado [como isotipo] por Barkley, 1962: 37; segundo paso de lectotipificación, G 00108089 [foto!], aquí designado; isolectotipos $\mathrm{B}$ [destruido], foto serie Field Museum 13177!, G 00108088 [foto!], G 00108090 [foto!]).

= Quebrachia morongii Britton, Ann. New York Acad. Sci. 7: 77. 1892. Tipo: Paraguay. Presidente Hayes, Pilcomayo River, 1888-1890, Morong 914 (Lectotipo NY, designado [como tipo] por Meyer \& Barkley, 1973: 214; segundo paso de lectotipificación, NY 00050811 [foto!]; isolectotipos G 00108091 [foto!], G 00108092 [foto!], GH 00049103 [foto!], MICH 1115716 [foto!], MO 260509 [foto!], NY 00050812 [foto!], PH 00023575 [foto!], US 00930530 [foto!], US 00095857 [foto!]); Kerr 55 (Sintipo, K 000572507 [foto!]).

= Schinopsis balansae Engl. var. pendula Tortorelli, Revista Argent. Agron. 10: 275, lám. 20. 1943. Tipo: Argentina. Chaco, Quitilipi, Legua 46, zona A, XII-1941, Tortorelli 391a (Holotipo LP 002741!; isotipo SI 000723!).

Al describir S. balansae, Engler (1885) citó como material original al ejemplar Balansa 3188, sin mencionar el herbario donde está depositado. Posteriormente Barkley (1962) mencionó con el status de "isotipo" a un espécimen depositado en G, y así lo designó (de hecho) como lectotipo del nombre S. balansae (Prado et al., 2015). Como en G hay tres cartulinas, se designa aquí, en un segundo paso de lectotipificación (Art. 9.17, Mc Neill et al., 2012), al ejemplar G 00108089. En este último espécimen aparece un determinavit de puño y letra de Barkley donde indica que es el tipo de la especie.

De la misma forma, al describir Quebrachia morongii, Britton (1892) citó como material original al ejemplar Morong 914, sin mencionar el herbario donde está depositado. Posteriormente Meyer \& Barkley (1973) mencionaron con el status de "tipo" a un espécimen depositado en NY, y así lo designaron (de hecho) como lectotipo del nombre $Q$. morongii (Prado et al., 2015). Como en NY hay dos cartulinas, se designa aquí, en un segundo paso de lectotipificación (Art. 9.17, Mc Neill et al., 2012) al ejemplar NY 00050811, porque se considera que en éste se expresan claramente todas las características de la especie mencionadas en el protólogo.

2. Schinopsis boqueronensis Mogni \& Oakley, Phytotaxa 175: 142. 2014. Tipo: Paraguay. Presidente Hayes, Ruta Trans-chaco, entre Pioneros y 25 Leguas, a la derecha del camino en dirección oeste-este, $22,73^{\circ} \mathrm{S} 59,69^{\circ} \mathrm{W}, 16-\mathrm{XII}-2011$, Vera et al. 4240 (Holotipo FCQ 51246!; isotipos CTES!, UNR!).

En varias ocasiones $S$. boqueronensis ha sido confundida morfológicamente con $S$. heterophylla (Muñoz, 1990; Navarro et al., 2006) debido, fundamentalmente, a que comparten el carácter de poseer tanto hojas simples como compuestas en un mismo individuo (Mogni et al., 2014). Estos dos taxones se diferencian principalmente por el patrón de nerviación, aspecto y morfología de la lámina. En S. boqueronensis las hojas son brillantes en la cara adaxial, oblongo-ovadas, con ápice emarginado, obtuso a sub-agudo y la base raramente lobada, y las venas secundarias divergen de la nervadura principal con un ángulo entre $60^{\circ}-90^{\circ}$; mientras que en $S$. heterophylla las hojas son glaucas, generalmente oblongo-lanceoladas con el ápice agudo a emarginado y la base frecuentemente lobada, y las venas secundarias divergen de la nervadura principal con un ángulo $55^{\circ}-60^{\circ}$. Adicionalmente, la presencia de restos estigmáticos en los frutos de $S$. boqueronensis y no en $S$. heterophylla es un carácter de importancia taxonómica.

3. Schinopsis brasiliensis Engl., en Martius, Fl. Bras. 12(2): 404, tab. 87. 1876. Tipo: Brasil. "Habitat in prov. Bahiae silvis Catingas", Martius s.n. (Lectotipo, aquí designado, M 0210719 [foto!]; isolectotipos M 0210721 [foto!], M 0210722 [foto!]).

= Schinopsis brasiliensis Engl. var. glabra Engl., en Martius, Fl. Bras. 12(2): 404. 1876. Schinopsis glabra (Engl.) F. A. Barkley \& T. Mey. Lilloa 33: 219. 1973. Tipo: Brasil. Bahia, Serra Jacobina, 6-XI-1839, Blanchet 2532 (Lectotipo, aquí designado, G 00237065 [foto!]; isolectotipos 
B [destruido], foto serie Field Museum 23184!, K 000572509 [foto!]).

El material original de $S$. brasiliensis depositado en M (Martius s.n.) consta de tres cartulinas con fragmentos que pertenecen supuestamente al mismo individuo, pero al no haber certeza de ello se designa al ejemplar M 0210719 como lectotipo del nombre de la especie, ya que se considera que en éste se expresan claramente las características mencionadas en el protólogo.

Al describir la variedad glabra de S. brasiliensis, Engler (1876) citó como material original al ejemplar Blanchet 2532, sin mencionar el herbario donde está depositado. Además, dicho autor ratifica esto en el tratamiento de la familia Anacardiaceae en la "Monographie Phanerogamarum" (Engler, 1883). Barkley (1962) y Meyer \& Barkley (1973) afirmaron erróneamente que el tipo de esta entidad es el ejemplar Martius 731 depositado en M. Sin embargo, el ejemplar Blanchet 2532 citado por Engler en el protólogo debe ser considerado como el tipo del nombre del taxón en cuestión (Art. 9.1, Mc Neill et al., 2012). De esta manera, se designa aquí como lectotipo del nombre $S$. brasiliensis var. glabra al ejemplar G 00237065, porque se considera que en éste se expresan claramente las características mencionadas en el protólogo.

4. Schinopsis cornuta Loes., Meded. RijksHerb. 27: 86. 1915. Tipo: Bolivia. Santa Cruz, Cordillera, Charagua, 800-860 m, XII-1910, Herzog 1148 (Lectotipo G 00237064 [foto!], designado [como tipo] por Barkley, 1962: 57; isolectotipos B [destruido], foto serie Field Museum 13178!, L 0726264 [foto!], Z 000000474 [foto!]).

Al describir S. cornuta, Loesener (1915) citó dos sintipos (ambos colectados por Herzog) como materiales originales de la nueva especie. Posteriormente Barkley (1962) al mencionar uno de estos ejemplares como tipo y especificar el herbario donde se encuentra, designó de hecho (Prado et al., 2015) como lectotipo del nombre $S$. cornuta al ejemplar Herzog 1148 depositado en G.

5. Schinopsis heterophylla Ragonese \& J. A. Castigl., Revista Invest. Agric. 1: 98. 1947. Tipo: Argentina. Chaco, Dpto. Com. Fernández, Pres. Roque Sáenz Peña, 1-III-1946, Ragonese
\& Castiglioni s.n. [ex herbario de la Dirección Forestal $\mathrm{N}^{\circ}$ 1957] (Lectotipo, aquí designado, BAB 00000038 [foto!]; isolectotipos BA 57983 [foto!], BAB 00000039 [foto!], BAB 00000040 [foto!], BAB 00000050 [foto!], CTES 0000752!).

El material original de $S$. heterophylla actualmente depositado en BAB (Ragonese \& Castiglioni s.n., ex herbario de la Dirección Forestal $N^{\circ}$ 1957) consta de cuatro cartulinas con ramas que aparentemente provienen del mismo individuo, aunque no hay datos adicionales que lo aseguren. Sin embargo, en uno de los ejemplares (BAB 00000038) parte de los datos en la etiqueta son manuscritos del propio Ragonese (R. Fortunato, com. pers.), uno de los autores de la especie, y se corresponde fielmente con el protólogo por lo cual se lo designa aquí como lectotipo.

6. Schinopsis lorentzii (Griseb.) Engl., Bot. Jahrb. Syst. 1: 46. 1880. $\equiv$ Loxopterygium lorentzii Griseb., Pl. Lorentz. 67: 115. 1874. Quebrachia lorentzii (Griseb.) Griseb., Abh. Königl. Ges. Wiss. Göttingen 24: 95. 1879; Symb. Fl. Argent. 95. 1879. Tipo: Argentina. Santiago del Estero, XII1871, Lorentz 24 (Lectotipo GOET 000101 [foto!], designado por Flores et al., 2013: 32; isolectotipos CORD 00004904 [foto!], LIL 001681!).

= Schinopsis haenkeana Engl., en Martius, Fl. Bras. 12(2): 406. 1876. Tipo: Perú. "Habitat in Peruvia", Häenke s.n. (Holotipo M 0212196 [foto!], foto serie Field Museum 20747!). Bolivia. Chuquisaca, Campero, Mizque, 2020 m, 15-II1967, Steinbach 683 (Epitipo, aquí designado, CTES 320325!; isoepitipos MO 1902013 [foto!], US, GH).

= Schinopsis marginata Engl., en A. DC. \& C. DC., Monogr. Phan. 4: 464. 1883. Schinopsis lorentzii (Griseb.) Engl. var. marginata (Engl.) Cabrera, Revista Mus. La Plata, Secc. Bot. 2: 54. 1938. Tipo: Argentina. Córdoba, al norte de la Sierra de Achala en las orillas del río Pinto, 8-III1877, Hieronymus 661 (Lectotipo, aquí designado, CORD 00003210 [foto!]; isolectotipos, GOET [foto!], F [foto!], NY, US).

= Aspidosperma quebracho-colorado Schltdl., Bot. Zeitung (Berlín) 19: 139. 1861. Schinopsis quebracho-colorado (Schltdl.) F. A. Barkley \& T. Mey. Bol. Soc. Argent. Bot. 3: 156. 1950. 
Algunos autores consideran a $S$. haenkeana como un taxón dudoso (Hunziker, 1998; Muñoz, 2000; Flores et al., 2013). Por ejemplo, Hunziker (1998) para sostener que el nombre debería ser relegado como dudoso se basó en el siguiente comentario del botánico Alberto Castellanos (según Sayago, 1969): "Sch. haenkeana es pediófila, es decir de las llanuras, como lo es Sch. lorentzii. Según creo, es la especie del chaco boliviano (en noviembre pasado lo encontré en el chaco brasileño). Es muy diferente de Sch. marginata de la Sierra Grande (Córdoba)...". Sin embargo, es muy probable que Castellanos se haya referido en realidad a Schinopsis brasiliensis, entidad muy común en la Chiquitanía Boliviana (Navarro \& Maldonado, 2002) y en el sudoeste de Brasil (Pinto, 1985; Prado et al., 1992), por lo que sus afirmaciones no deberían ser consideradas como algo concluyente. Por otra parte, Flores et al. (2013) hacen alusión al origen geográfico (Perú) del material original coleccionado por T. Haenke y remarcan que para dicho país no se cita la especie. Sin embargo, es probable que el material original de $S$. haenkeana haya sido coleccionado en Bolivia, donde sí se ha citado la especie (Vargas Salazar, 1993; Navarro \& Maldonado, 2002), ya que para la época en que el mencionado naturalista hacía sus colectas, dicho país se conocía como 'Alto Perú' (Gómez Méndez, 2003). Asimismo existen numerosas evidencias de que Haenke coleccionó en Bolivia (aproximadamente desde el año 1794) y que también residió allí, hasta que falleció en 1816, en la ciudad de Cochabamba (Funk \& Mori, 1989). Otra consideración de Flores et al. (2013) hace referencia a que el material original (holotipo) de $S$. haenkeana depositado en $\mathrm{M}$ se encuentra muy dañado y que por lo tanto no permite su identificación correcta. Sin embargo, al estudiar la colección de fotografías de tipos del Field Museum se encontró que la foto $\mathrm{n}^{\circ} 20747$, tomada al espécimen Haenke s.n. en el año 1932, se corresponde con el material original de $S$. haenkeana actualmente disponible en M (Fig. 1). En ésta hay tres hojas y una rama (si bien corta) representativa y con la inflorescencia típica, que sí se condicen con lo descripto por Engler (1876) en el protólogo. Por todo lo expuesto se considera que Schinopsis haenkeana Engl. es un nombre correcto y para reforzar su identidad taxonómica, se designa

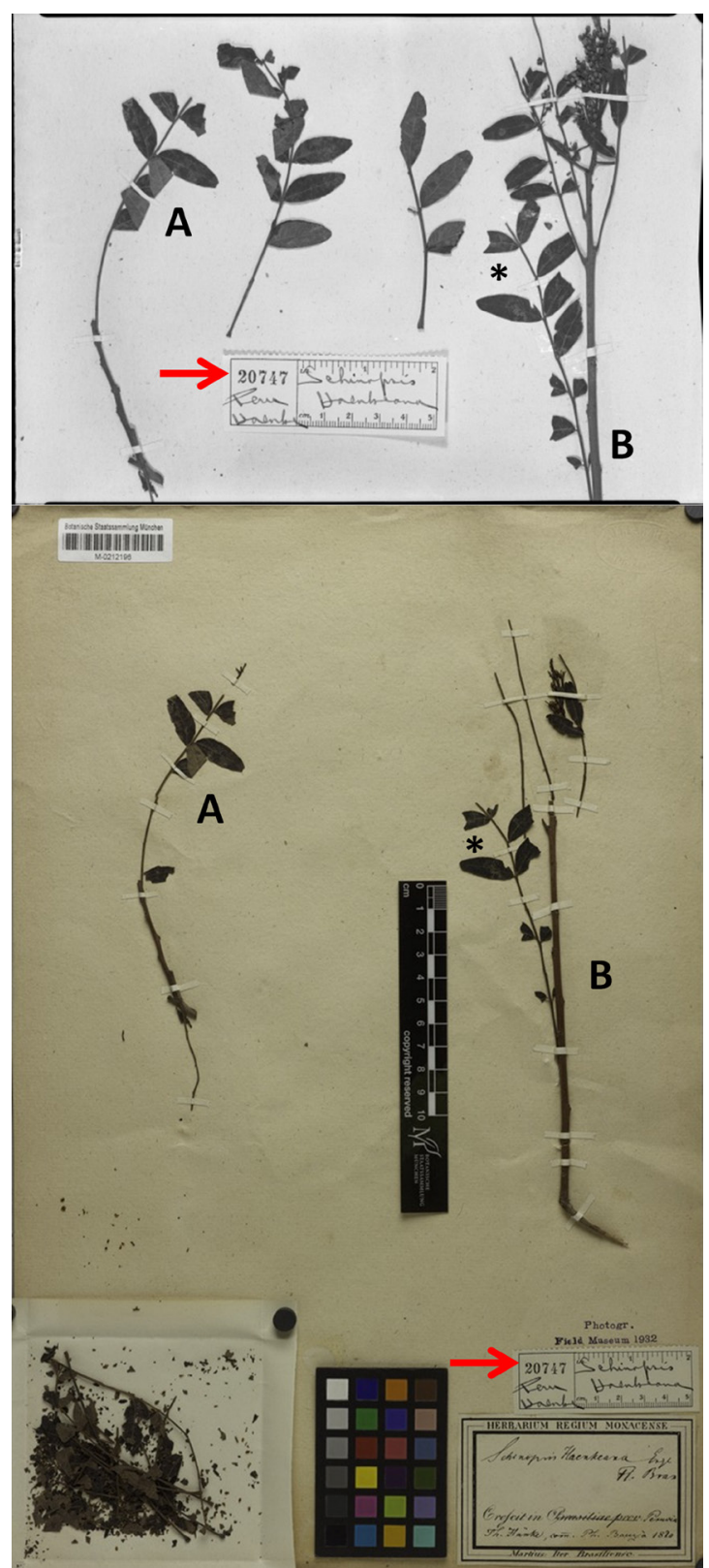

Fig. 1: Espécimen tipo de S. haenkeana. Fotografía $n^{\circ} 20747$ de 1932 (imagen superior), y la inferior el mismo ejemplar en M. Las letras A y B indican las partes del material original visibles tanto en la antigua fotografía (superior), como en la actual (inferior). Los asteriscos señalan segmentos de referencia para una rápida comparación. Las flechas indican la etiqueta original colocada por el fotógrafo. 
aquí el epitipo correspondiente (Art. 9.8, Mc Neill et al., 2012).

Flores et al. (2013) afirman que el lectotipo de $S$. marginata fue designado por Hunziker (1998); sin embargo esto es erróneo, ya que en su trabajo dicho autor citó textualmente: "Schinopsis marginata Engler, Typus: Hieronymus 661 (CORD, GOET)", sin hacer mención del herbario al cual corresponde el lectotipo. De acuerdo al Art. 9.22 del ICBN (Mc Neill et al., 2012), para que una lectotipificación sea llevada a cabo el autor debe mencionar específicamente el herbario donde está depositado el espécimen, y esto último no ocurrió en este caso.

Es de destacar que en el caso de que la especie conocida vulgarmente como "orco quebracho" (Hunziker, 1998), "quebracho serrano" o "quebracho colorado del cerro" (Muñoz, 2000) sea considerada como independiente de Schinopsis lorentzii ("quebracho colorado santiagueño", "coronillo"), el nombre correcto para ese taxón deberá ser Schinopsis haenkeana, pasando $S$. marginata a formar parte de su sinonimia (Art. 11.1, Mc Neill et al., 2012).

Con respecto al binomio $S$. quebracho-colorado se acepta el criterio de que debe ser abandonado y considerado un taxón dudoso (Hunziker, 1998; Muñoz, 2000; Flores et al., 2013). Sin embargo, debido a que en varias oportunidades ha sido utilizado, tanto en tratamientos florísticos (v.g. Muñoz, 1990) como en bibliografía no taxonómica (v.g. Spichiger et al., 1991) se recomienda citarlo en la sinonimia de $S$. lorentzii.

7. Schinopsis peruviana Engl., en Martius, Fl. Bras. 12(2): 405, t. 86, fig. 2. 1876. Tipo: Perú. San Martín, prope Tarapoto, Peruviae orientalis, 1855/6, Spruce 4549 (Lectotipo, aquí designado, $\mathrm{K} 000110222$ [foto!]; isolectotipos K 000110334 [foto!], BR 000000521259 [foto!], C 10005511 [foto!], F 871730 [foto!], F 937856 [foto!], G, GH 00049162 [foto!], GH 00049163 [foto!], MPU 021091 [foto!], NY 00050879 [foto!], W 50879 [foto!]).

Al describir S. peruviana, Engler (1876) citó como material original al ejemplar Spruce 4549, sin mencionar el herbario donde está depositado. Posteriormente Barkley (1962) y Meyer \& Barkley
(1973) afirmaron que existían isotipos en los herbarios C, F, G, GH y NY, aunque sin indicar dónde se encuentra el supuesto original. Como estos autores hacen mención a más de un herbario, es imposible en este caso, considerar que hubo una lectotipificación no intencional (Prado et al., 2015). De acuerdo a esto último, se designa aquí como lectotipo al ejemplar K 000110222 , porque se considera que en éste se expresan claramente todas las características de la especie mencionadas en el protólogo.

\section{Agradecimientos}

A las autoridades y curadores de los herbarios consultados (BA, BAB, CORD, CTES, FCQ, LIL, LP y SI), y a UNR por la licencia para examinar ejemplares tipo en forma digital (JStor Global Plants). Nuestro particular agradecimiento al Field Museum (F) y al Munich herbarium (M) por facilitar las imágenes del material original de $S$. haenkeana y por la autorización para su uso y publicación. A la Universidad Nacional de Rosario (UNR) y al Consejo Nacional de Investigaciones Científicas y Técnicas (CONICET). A Jefferson Prado por su invalorable asesoramiento. A Renée H. Fortunato y María Alexandra Monsalvo por facilitar imágenes de alta calidad del material tipo depositado en el Instituto de Recursos Biológicos del INTA Castelar (BAB). A tres revisores anónimos por sus valiosas contribuciones al manuscrito.

\section{Bibliografía}

BARKLEY, F. A. 1962. Anacardiaceae: Rhoideae: Schinopsis. Proc. Iraqi Sci. Soc. 5: 44-69.

BRITTON, N. L. 1892. Plants Collected in Paraguay. Ann. New York Acad. Sci. 7: 45-280.

CABRERA A. L. 1938-1939. Revisión de las Anacardiáceas austroamericanas. Rev. Mus. La Plata Secc. 2: 3-64.

DE CANDOLLE, A. \& C. DE CANDOLLE (eds.). 1883. Monographie Phanerogamarum. Vol. IV. Masson, Paris.

ENGLER, H. G. F. 1876. Anacardiaceae. En: MARTIUS, C. F. P. von (ed.), Flora Brasiliensis, vol. 12, part 2, pp. 367-418. F. Fleischer, Monachii \& Lipsiae. 
ENGLER, H. G. F. 1885. Eine neue Schinopsis. Bot. Jahrb. Syst. 6: 286.

FIELD MUSEUM. Field Museum of Natural History (Botany) Seed Plant Collection. Berlin Negatives. Disponible en: https://www.fieldmuseum.org/ node/5186 y http://www.gbif.org/dataset/90c853e656bd-480b-8e8f-6285c3f8d42b [Acceso: 04 de marzo de 2016].

FLORES, C., M. ZAPATER \& S. SÜHRING. 2013. Identidad taxonómica de Schinopsis lorentzii y Schinopsis marginata (Anacardiaceae). Darwiniana, nueva serie 1: 25-38.

FUNK, V. A. \& S. A. MORI. 1989. A bibliography of plant collectors in Bolivia, Smithson. Contrib. Bot. 70: 1-20.

GÓMEZ MÉNDEZ, S. O. 2003. Atlas de Historia de América. Limusa, Noriega editores, México.

HUNZIKER, A. 1998. Los nombres científicos correctos de los "quebrachos colorados" (Schinopsis, Anacardiaceae) del centro y noroeste de Argentina. Kurtziana 26: 55-64.

JSTOR GLOBAL PLANTS. Disponible en: http://plants. jstor.org/ [Acceso: 14 de marzo de 2016].

JUDD, W., C. CAMPBELL, E. KELLOGG, P. STEVENS \& M. DONOGHUE. 1999. Plant Systematics: A Phylogenetic Approach. $2^{\text {nd }}$ ed. Sinauer Associates, Sunderland.

LOESENER, L. E. T. \& T. C. J. HERZOG. 1915. Herzog's bolivianische Pflanzen II: Anacardiaceae. Mededeelingen van's Rijks-Herbarium 27: 84-88.

MCNEILL, J., F. R. BARRIE, W. R. BUCK, V. DEMOULIN, W. GREUTER, D. L. HAWKSWORTH, P. S. HERENDEEN, S. KNAPP, K. MARHOLD, J. PRADO, W. F. PRUD'HOMME VAN REINE, G. F. SMITH, J. H. WIERSEMA \& N. J. TURLAND (eds.). 2012. International Code Nomenclature for algae, fungi and plants (Melbourne Code). Reg. Veg. 154. Koeltz Scientific Books, Königstein.

MCNEILL, J. 2014. Holotype specimens and type citations: General issues. Taxon 63: 1112-1113.

MEYER, T. \& F. A. BARKLEY. 1973. Revisión del género Schinopsis (Anacardiaceae). Lilloa 33: 207-257.

MOGNI, V. 2015. Estudio Filogenético y Biogeográfico del género Schinopsis (Anacardiaceae). Tesis Doctoral, Facultad de Ciencias Agrarias, Universidad Nacional de Rosario, Rosario, Argentina, pp. 250.

MOGNI, V., L. OAKLEY, M. VERA JIMÉNEZ \& D. E. PRADO. 2014. A new tree species of Schinopsis (Anacardiaceae) from Paraguay and Bolivia. Phytotaxa 175: 141-147.

MUÑOZ, J. D. 1990. Anacardiaceae. En: SPICHIGER, R. \& L. RAMELLA (eds.) Flora del Paraguay 14, pp. 5-84. Missouri Botanical Garden \& Conservatoire et Jardin Botaniques de la Ville de Geneve.

MUÑOZ, J. D. 2000. Anacardiaceae. En: HUNZIKER, A. T. (ed.), Flora Fanerogámica Argentina 65: 1-28.
NAVARRO, G. \& M. MALDONADO. 2002. Geografia Ecológica de Bolivia. Vegetación y Ambientes Acuáticos. Centro de Ecología Simón I. PatiñoDepartamento de Difusión, Cochabamba.

NAVARRO, G., J. MOLINA \& L. PÉREZ DE MOLAS. 2006. Classification of the forests of the northern Paraguayan Chaco. Phytocoenologia 36: 473-508.

PELL, S. K. 2004. Molecular systematics of the cashew family (Anacardiaceae). PhD Dissertation, Louisana State University, Baton Rouge, pp.193.

PINTO, G. C. P. 1985. Schinopsis brasiliensis Engl. na comunidade florística do Pantanal. Anais do XXXIII Congresso Nacional de Botânica, Maceió, 1982 (ed. Sociedade Botânica do Brasil), pp. 49-51. EMBRAPA, Brasilia.

PRADO, D. E. 1993a. What is the Chaco vegetation in South America? I. A review. Candollea 48: 145-172.

PRADO, D. E. 1993b. What is the Chaco vegetation in South America? II. A redefinition. Candollea 48: 615629.

PRADO, D. E. 2000. Seasonally dry forests of tropical South America: from forgotten ecosystems to a new phytogeographic unit. Edinburgh J. Bot. 57: 437-461.

PRADO, D. E., P. E. GIBBS, A. POTT \& V. J. POTT. 1992. The Chaco-Pantanal transition in southern Mato Grosso, Brasil. En: FURLEY, P. A., J. PROCTOR \& J. A. RATTER (eds.), Nature \& Dynamics of ForestSavanna Boundaries, pp. 451-470. Chapman \& Hall, London.

PRADO, J., R. Y. HIRAI \& R. C. MORAN. 2015. Proposals concerning inadvertent lectotypifications (and neotypifications). Taxon 64: 651.

RAGONESE, A. \& J. CASTIGLIONI. 1947. Nueva especie del género Schinopsis y área geográfica de las especies argentinas. Rev. Invest. Agr. 1: 93-100.

SAYAGO M. 1969. Estudio Fitogeográfico del Norte de Córdoba. Bol. Acad. Nac. Cienc. 46: 123-427.

SPICHIGER, R.; L. RAMELLA; R. PALESE \& F. MERELES 1991. Proposición de leyenda para la cartografía de las formaciones vegetales del Chaco Paraguayo. Contribución al estudio de la flora y de la vegetación del Chaco III. Candollea 46: 541-564.

THIERS, B. 2013. Index Herbariorum: A global directory of public herbaria and associated staff. New York Botanical Garden's Virtual Herbarium. Disponible en: http://sweetgum.nybg.org/ih/ [Acceso: 14 de marzo de 2016].

VARGAS SALAZAR, E. 1993. Anacardiaceae. En: KILLEEN, T., E. GARCÍA \& S. BECK (eds.), Guía de árboles de Bolivia, pp. 83-97. Herbario Nacional de Bolivia-Missouri Botanical Garden. Quipus SRL, La Paz.

Recibido el 28 de junio de 2016, aceptado el 20 de octubre de 2016. 